\title{
Microwave Effect on Curing of Waterborne Polyurethane
}

\author{
Hoikwan Lee, ${ }^{*}$ Chris. Y. Fang, Carlo. G. Pantano, and Wonho Kang ${ }^{\dagger}$ \\ Materials Research Institute, The Pennsylvania State University, University Park, PA 16802, USA. *E-mail: hoikwanlee@gmail.com \\ 'Department of Advance Materials Science and Engineering, Dankook University, Cheonan 330-714, Korea \\ Received August 25, 2010, Accepted January 17, 2011
}

\begin{abstract}
Spin-coated waterborne polyurethane to protect glass surface from environmental attacks was cured by using microwave heating. The effect of microwave heating on the reaction kinetics, chemical durability, and transmittance of polyurethane was investigated. In comparison to the conventional heating the results show that the microwave heating substantially accelerates the curing process of waterborne polyurethane and the total time for the completion of the reaction is only $1 / 7$ of that in the conventional process. The microwave cured sample showed an excellent caustic resistance compared to conventional cured one. It means that microwave heating produces dense structure during curing process. The dense structure does not affect to the transmittance in the visible region.
\end{abstract}

Key Words : Curing, Microwave heating, Polyurethane, Coating, Caustic resistance

\section{Introduction}

For effective materials processing (i.e. sintering, annealing, and curing) and to improve materials properties, the application of the microwave heating has been investigated because it has the potential to provide for uniform, rapid heating, and to reduce the processing time for polymers and ceramics, which are materials with low thermal conductivities. $^{1}$

In microwave heating (MW denotes after this), the energy is directly delivered to the materials through molecular/ion interaction with the electromagnetic field while the energy in conventional heating ( $\mathrm{CV}$ denotes after this) is transferred to the materials through the convection, conduction, and radiation of heat from the surface to the inside. Microwave heating can produce rapid and uniform heating in materials due to its volumetric heating. Another characteristic of microwave heating is utilized for the selective heating in the multi-component materials. The interaction between microwave and materials depends on the molecular structure. So if a sample was combined with several materials having different dielectric properties, the microwaves will selectively couple with a higher loss material. Because of this reason, microwave heating may also be possible to initiate chemical reactions not possible in conventional process through the selective heating of the reactants. ${ }^{1,2}$

Microwave activation of polymerization reactions at 2.45 $\mathrm{GHz}$ first came into use in the early 1970 's. ${ }^{3}$ Substantial investigations have been done since then. Some significant improvement in properties of the cured polyurethanes(PU denotes after this) with microwave has been reported. ${ }^{4-6} \mathrm{PU}$ is one of the important organic materials with ever increasing applications due to their excellent adhesion to most materials, excellent scratch resistance, excellent chemical stability etc. In the connection with environmental concern, the waterborne polyurethanes are more and more popular compared to the solvent-borne polyurethanes. Film coating is a popular form of PU applications. A PU coating can also effectively improve mechanical performance and chemical stability of the coated substrate and help protect the coated articles. $^{7-9}$

In this study, therefore microwave process was studied for the curing of waterborne polyurethane spin-coated on glass substrates and the effects of microwave processing on the reaction kinetics, chemical stability, and optical property of polyurethane coating on glass substrates were evaluated.

\section{Experimental}

There are many PU systems. Some are single component, the others are two or multi-components. Most PU systems set during thermal treatment. Isocyanate group, $\mathrm{NCO}$, is the most important functional group in the PU materials. NCO groups can readily react with polyols. The isocyanate groups can be chemically blocked by compounds such as caprolactam, butanone oxime, phenol, or dimethylpyrazole. At room temperature, the blocked polyisocyanates do not react with polyols at any appreciable rate. At elevated temperatures, the blocked polyisocyanate liberates the blocking agent, which may leave the film, and the polyisocyanate reacts with the polyol.

Bayer RR6895, which is a 2-component PU with chemically blocked $\mathrm{NCO}$, is recommended for conventional curing conditions at $443 \mathrm{~K}$ for $30 \mathrm{~min}$ in air after drying, was used for the microwave processing experiment. Component 1 contains Bayhydur VP LS 2240 (crosslinking agent, 35\% solid, water-dispersed blocked aliphatic polyisocyanate resin based on dicyclohexylmethane diisocyanate, H12MDI, dissolved in water/MPA/xylene, 56:4.5:4.5) and the Bayhydrol VP LS 2239(water-thinnable hydroxyl-bearing polyurethane dispersion). Component 2 includes dipropylene glycol and 2 silanes $\left(0.2 \%\right.$ each) as adhesion promoters, Silquest ${ }^{\circledR}$ A- 
189 Silane (Gamma-mercaptopropyltrimethoxysilane, CAS: 56938-96-6, USA) and Dynasylan ${ }^{\circledR}$ AMEO (3-Aminopropyltriethoxysilane, CAS: 919-30-2, USA). The polyurethane reaction in Component 1 of this system is as the following:<smiles>[R]OC(=O)NNC(=O)O[R17]([H])Br</smiles>

“2240" "2239" polyurethane blocking agent

The two components were mixed together at room temperature and then the mixed precursor was spin coated on sodalime silicate glass at $5 \mathrm{~K} \mathrm{rpm}$ for $60 \mathrm{~s}$ using a static dispenser program. The samples were then cured by a microwave process for 1-2 min in a thermally insulated package that was preheated to about $170{ }^{\circ} \mathrm{C}$ with microwave. In order to assist the samples heated uniformly in the microwave cavity, an insulation package with $\mathrm{SiC}$ as the microwave suceptor was used and a Teflon sample holder slotted with PU coated glass was located in the center of the insulated casket. The average thickness of the cured PU coating was about $10 \mu \mathrm{m}$. For comparison, the same curing process was also carried out with a conventional electrical furnace.

For the kinetic study, the Infrared absorption spectras (FTIR, Bruker Optics Inc., Billerica, MA) of the samples were obtained during the drying/curing process to trace the change in the characteristic band at $2270 \mathrm{~cm}^{-1}$ (NCO) and to monitor the reaction between the $\mathrm{NCO}$ and $\mathrm{ROH}$-groups to form polyurethane. The caustic resistance of the PU coatings was evaluated under accelerated conditions by soaking the cured sample in a $2 \% \mathrm{NaOH}$ solution at $85 \pm 2{ }^{\circ} \mathrm{C}$ in an oil bath. The transmittance of the PU coated glass was measured with a UV-Visible spectrophotometer (CARY 2300, Varian, Texas). The microstructure of cured polyurethane was observed by Scanning electron microscopy (SEM, Hitachi S-300H, Hitachi High-Technologies, Tokyo).

\section{Results and Discussion}

Figure 1 shows the characteristic band at $2270 \mathrm{~cm}^{-1}$ (NCO) in the FT-IR spectra used to monitor the reaction between the $\mathrm{NCO}$ and $\mathrm{ROH}$-groups to form polyurethane as a function of the curing time. It is seen that this band becomes stronger at the beginning of process and gets weak after reaching maximum point.

Figure 2 presents the FT-IR intensity of the band corresponding to NCO. In the conventional processing, the curing process can be divided into 4 stages: the unlocking of $\mathrm{NCO}$ (1), reaction (2), intermediate stage (3), and final stage (4). In the microwave process, on the other hand, it was found that the rate of the NCO unlocking and reaction stages was 7 times of that in the conventional process. In addition, the rate of the final stage was about 23 times of that in the conventional process and the polymerization was almost completed. It should be noted that the microwave processing substantially enhanced the reaction kinetics and that the total
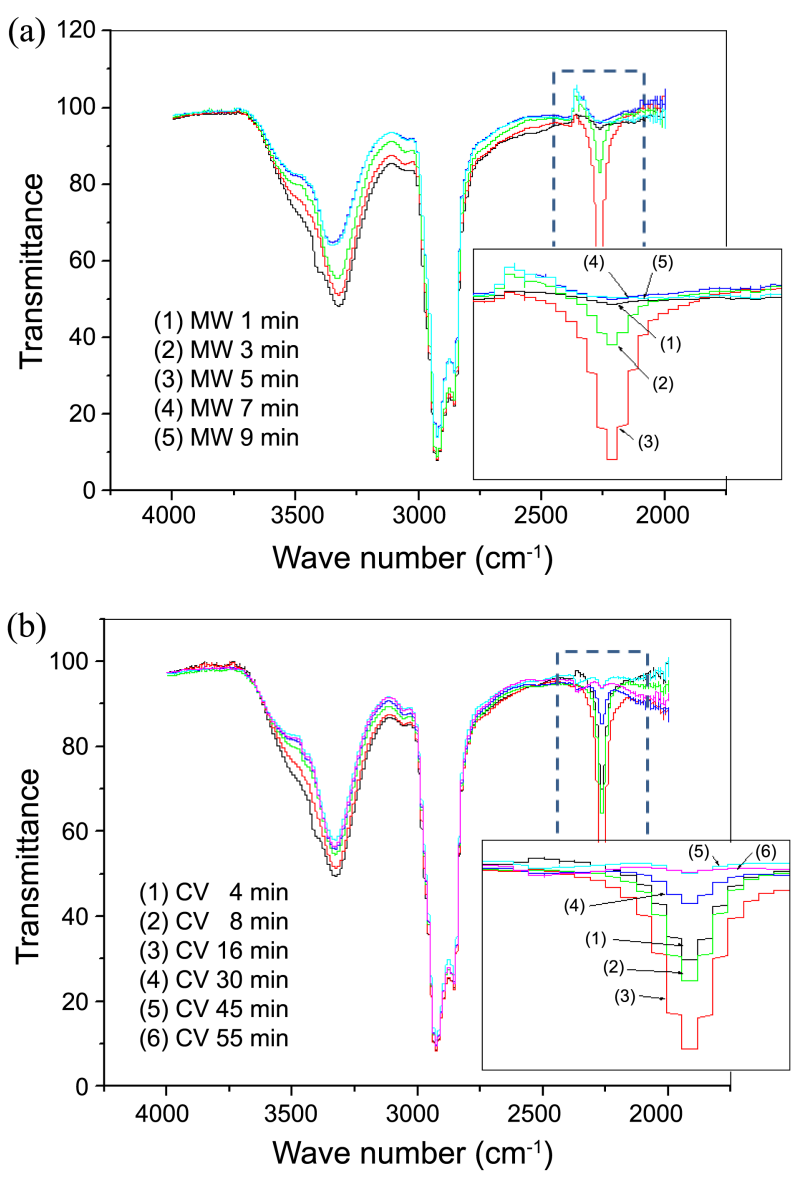

Figure 1. FT-IR spectras according to curing time (a) MW heating and (b) CV heating.

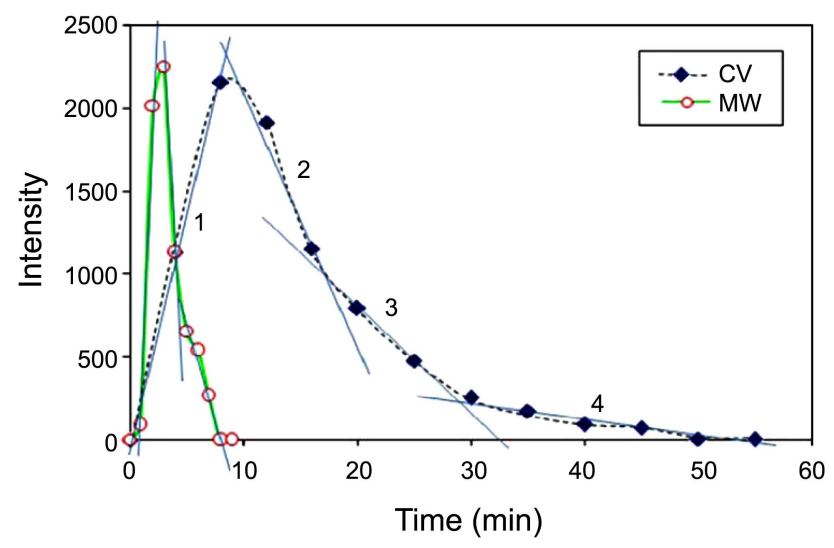

Figure 2. FT-IR intensity of NCO as a function time showing the reaction kinetics of $\mathrm{PU}$ by microwave processing and conventional processing at $170^{\circ} \mathrm{C}$.

time for the completion of the reaction was only $1 / 7$ of that in the conventional process.

Figure 3 shows the facture surfaces of microwave and conventional cured samples which survived the caustic test. The microwave sample in Figure 5(a) treated under harsh environmental condition for 21 days showed a excellent caustic resistance compared to the $\mathrm{CV}$ cured sample without forming bubbles or peeling of the coated PU. In case of CV 

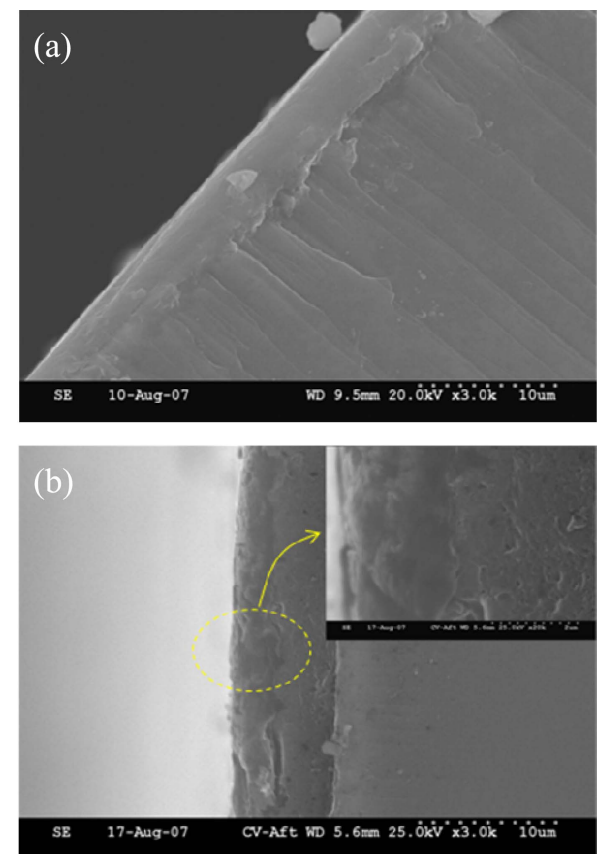

Figure 3. Fracture surface after caustic resistance test. (a) MW cured sample $\left(7 \mathrm{~min}\right.$. at $170{ }^{\circ} \mathrm{C}$ ) in $2 \% \mathrm{NaOH}$ solution Bath for 21 days. (b) $\mathrm{CV}$ cured sample $\left(30 \mathrm{~min}\right.$. at $\left.190{ }^{\circ} \mathrm{C}\right)$ in $2 \% \mathrm{NaOH}$ solution bath for 13 days.

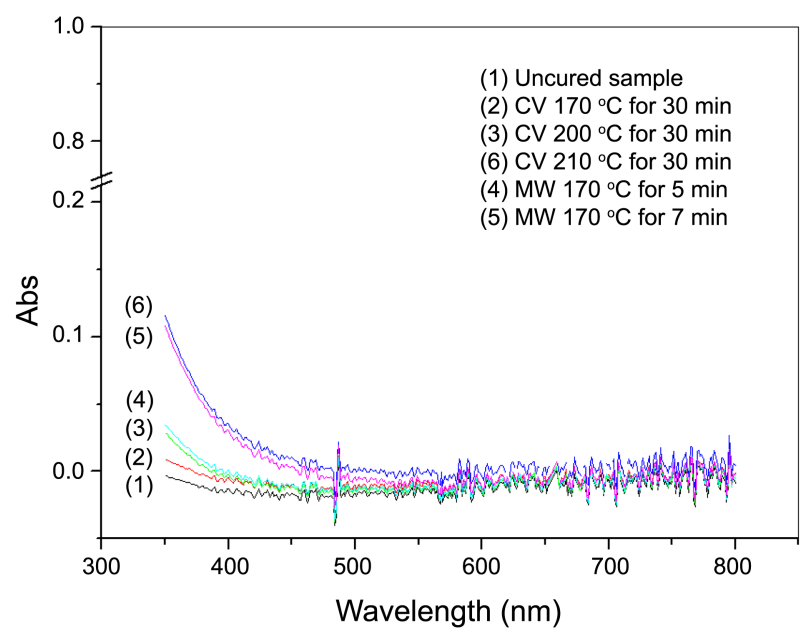

Figure 4. UV-vis spectras as a function of curing conditions.

cured sample in Figure 5(b) also showed a good adhesion without forming bubbles or peeling. The surface layer about $3 \mu \mathrm{m}$ thick was only damaged. Since the caustic resistance of the PU coating depends on its density, chemical stability, and interfacial adhesion to the substrate, the results imply that microwave processing considerably enhanced all of these aspects.

Figure 4 presents the absorption spectra after curing with the conventional and microwave processing. Air was used as a control sample. No significant absorption was observed in the UV-vis region and it was observed that the absorption at around $350 \mathrm{~nm}$ increased slightly when the samples were overcured.

The chemical resistance of the PU coated on glass allows it to be used to protect the glass surface in harsh environment. Currently many commercial glasses which have high alkali content are highly susceptible to corrosion due to the leaching of alkali ions from the glass surface when it comes into contact with water or humidity. This influences the appearance, optical transmittance, strength, and interface stability with other materials. For these reasons, the ability to control the absorption of water and organic materials through surface treatment or coatings is required to improve the performance and reliability of the glass surface, as well as to expand the field of applications of glasses.

The coating of PU and curing by microwave processing could be used as a coating precursor and a new curing method, in order to allow the glass to become highly densified and have good adhesion to the substrate.

\section{Conclusions}

In this work, microwave effect on curing of waterborne polyurethane coated on glass substrate was studied and the result was compared with that of the conventionally cured samples. The reaction kinetics of the PU was accelerated by 7-23 times depending on the specific stages and the caustic resistance of the microwave processed PU samples was improved. It implies that to this specific PU system, microwave processing effectively improved the properties related to density, moisture stability, chemical stability, and adhesion of the PU coating to the substrate. This study demonstrated the microwave heating has significant potential for a wide range of application in curing polymer and saving time (energy).

Acknoledgments. This research was supported by the Bayer Materials Science research fund.

\section{References}

1. Thostenson, E. T.; Chou, T. W. Composites: Part A 1999, 30, 1055.

2. Clark, D. E.; Gac, F. D.; Sutton, W. H. Ceramic Transactions 21; The American Ceramic Society: 1991.

3. Gourdenne, A.; Maassarani, A. H.; Monchaux, P.; Aussudre, S.; Thourel, L. Polym. Prepr. Am. Chem. Soc. Div. Polym. Chem. 1979, 20, 471 .

4. Gourdenne, A. High Performance Polymers 2005, 17, 425.

5. Jullien, H.; Valot, H. Polymer. 1985, 26, 506.

6. Marand, E.; Baker, H. R.; Graybeal, J. D. Macromolecules 1992, $25,2243$.

7. Wang, J.; Sha, J.; Yang, Q.; Wang, Y.; Yang, D. Mater. Res. Bull. 2005, 40, 1551.

8. Zhang, J.; Lin, J.; Song, H. S.; Elssfah, E. M.; Liu, S. J.; Luo, J. J.; Ding, X. X.; Tang, C.; Qi, S. R. Mater. Lett. 2006, 60, 3292.

9. Cheng, C.; Ding, X. X.; Shi, F. J.; Cheng, Y.; Huang, X. T.; Qi, S. R.; Tang, C. J. Cryst. Growth 2004, 263, 600. 